\title{
Growth Mechanisms of Nanostructured Titania in Turbulent Reacting Flows
}

\author{
Sean C. Garrick \\ Department of Mechanical Engineering, University of Minnesota, 111 Church Street SE, Minneapolis, MN 55455-0111, USA \\ Correspondence should be addressed to Sean C. Garrick; sgarrick@umn.edu
}

Received 5 June 2015; Accepted 22 July 2015

Academic Editor: Lin-Hua Xu

Copyright ( 2015 Sean C. Garrick. This is an open access article distributed under the Creative Commons Attribution License, which permits unrestricted use, distribution, and reproduction in any medium, provided the original work is properly cited.

\begin{abstract}
Titanium dioxide (titania) is used in chemical sensors, pigments, and paints and holds promise as an antimicrobial agent. This is due to its photoinduced activity and, in nanostructured form, its high specific surface area. Particle size and surface area result from the interplay of fluid, chemical, and thermal dynamics as well as nucleation, condensation and coagulation. After nucleation, condensation, and coagulation are the dominant phenomena affecting the particle size distribution. Manufacture of nanostructured titania via gas-phase synthesis often occurs under turbulent flow conditions. This study examines the competition between coagulation and condensation in the growth of nanostructured titania. Direct numerical simulation is utilized in simulating the hydrolysis of titanium tetrachloride to produce titania in a turbulent, planar jet. The fluid, chemical, and particle fields are resolved as a function of space and time. As a result, knowledge of titania is available as a function of space, time, and phase (vapor or particle), facilitating the analysis of the particle dynamics by mechanism. Results show that in the proximal region of the jet nucleation and condensation are the dominant mechanisms. However once the jet potential core collapses and turbulent mixing begins, coagulation is the dominant mechanism. The data also shows that the coagulation growth-rate is as much as twice the condensation growth-rate.
\end{abstract}

\section{Introduction}

High rate synthesis of nanoparticles from vapor requires operation in the turbulent flow regime [1]. The variety of length and time scales present in turbulent multiphase flows makes them not very amenable to physical observation or analysis. This is especially true in the early stages of the nanoparticle formation and growth processes [2]. Computational fluid dynamics (CFD) has been developed for many years and a variety of numerical techniques have been developed and utilized for studying particle dynamics [310]. CFD has enabled engineers to achieve their goals more rapidly and cost effectively [11]. It has also become an effective tool for understanding physicochemical dynamics.

Nakaso et al. [12] modeled the titania nanoparticle growth of both agglomerates and primary particles simultaneously by using spatial zero-dimensional fluid dynamics. Johannessen et al. [13] combined CFD with a mathematical model for the particle dynamics to compare with experimental data from the synthesis of titanium dioxide particles in diffusion flames. Tsantilis et al. [5] used a moving sectional aerosol dynamics model accounting for gas-phase chemical reactions, coagulation, surface growth, and sintering with zerodimensional fluid dynamics to investigate flame synthesis of titania nanoparticles. Also one study by Moody and Collins [14] considered titania nanoparticle nucleation and growth in a turbulent "box" located near the center of the reactor via three-dimensional DNS coupled with moment method in particle dynamics. Researchers have also considered the effects of turbulence on particle growth. Strakey et al. [15] studied the role of turbulence on the characteristics of $\mathrm{TiO}_{2}$ powder made by $\mathrm{TiCl}_{4}$ oxidation and they found that the increased turbulence intensity narrowed the size distribution of the product powder indicating that the particle growth may be dominated by reactant mixing rather than by particleparticle collisions. These computations are quite compute intensive as they resolve all of the appropriate length and time scales. Garrick and his group studied the effects of turbulence on particle coagulation intensively and indicated that turbulence has a positive effect on the particle growth 
$[16,17]$. The effects of turbulence on nucleation have also been elucidated [18-20]. To obtain physically accurate data, simulations must be three-dimensional and must be modelfree. That is, the results must be obtained without the use of turbulence or subgrid scale models [21]. A review of the literature reveals a lack of the detailed information, especially via high-resolution direct simulation, on the interplay between the different mechanisms affecting nanoparticle growth.

In this work, the hydrolysis of titanium tetrachloride $\left(\mathrm{TiCl}_{4}\right)$ to produce titanium dioxide $\left(\mathrm{TiO}_{2}\right)$ is simulated via direct numerical simulation. The turbulent, reacting, multiphase flow is obtained by solving the Navier-Stokes equations in conjunction with transport equations for all of the relevant chemical species and a nodal approach is used to represent the particle field $[22,23]$. With the chemical and particle fields available as a function of space, time, and size, particle nucleation, condensation, and coagulation are illustrated individually. Additionally the two growth mechanisms are elucidated.

\section{Methodology}

The mass, momentum, and energy equations are solved to obtain the fluid velocity $u_{i}(\bar{x}, t)$, pressure $p(\bar{x}, t)$, density $\rho(\bar{x}, t)$, and the enthalpy $h(\bar{x}, t)$. These variables are governed by the following conservation equations:

$$
\begin{gathered}
\frac{\partial \rho}{\partial t}+\frac{\partial \rho u_{j}}{\partial x_{j}}=0, \\
\frac{\partial \rho u_{i}}{\partial t}+\frac{\partial \rho u_{i} u_{j}}{\partial x_{j}}=-\frac{\partial p}{\partial x_{i}}+\frac{\partial \tau_{i j}}{\partial x_{j}}, \\
\frac{\partial \rho h}{\partial t}+\frac{\partial \rho u_{j} h}{\partial x_{j}}=\frac{\partial}{\partial x_{j}}\left(\frac{k}{C_{p}} \frac{\partial h}{\partial x_{j}}\right),
\end{gathered}
$$

where $\tau_{i j}$ is the viscous stress tensor for a Newtonian fluid, $k$ is the coefficient of thermal conduction, and $C_{p}$ is the specific heat at constant pressure.

2.1. Chemical Transport. The fluid contains five chemical species, the transport of which is given by the conservation of species equations:

$$
\frac{\partial \rho Y_{m}}{\partial t}+\frac{\partial \rho u_{j} Y_{m}}{\partial x_{j}}=\frac{\partial}{\partial x_{j}}\left(\rho \mathscr{D}_{m} \frac{\partial Y_{m}}{\partial x_{j}}\right)+\dot{\omega}_{m},
$$

where $Y_{m}$ is the mass concentration of species $m$ and $\mathscr{D}_{m}$ is the diffusion coefficient of species $m$. The reactants, $\mathrm{TiCl}_{4}$ and water vapor $\left(\mathrm{H}_{2} \mathrm{O}\right)$, undergo an irreversible one-step, isothermal chemical reaction at a temperature of $300 \mathrm{~K}$ and atmospheric pressure, which produces titanium dioxide $\left(\mathrm{TiO}_{2}\right)$ and hydrochloric acid $(\mathrm{HCl})$ :

$$
\mathrm{TiCl}_{4}+2 \mathrm{H}_{2} \mathrm{O} \stackrel{k_{f}}{\longrightarrow} \mathrm{TiO}_{2}+4 \mathrm{HCl}
$$

The source term $\dot{\omega}_{m}$ in (2) represents the effects of chemical reaction, the rate of creation or consumption of species $m$.
The system is closed with the ideal gas equation of state, $p=$ $\rho R T$ and $R=\sum R_{m} Y_{m} / M W_{m}$, where $R_{m}$ and $M W_{m}$ are the gas constant and molecular weight of species $m$, respectively. The fluid temperature, $T$, is obtained using the enthalpy via $d h=C_{p} d T$. The fifth chemical species, nitrogen $\left(\mathrm{N}_{2}\right)$, does not participate in any chemical reactions and serves as the carrier gas.

2.2. Particle Field. The aerosol general dynamic equation (GDE) describes particle dynamics under the influence of various physicochemical phenomena, convection, diffusion, coagulation, surface growth, nucleation, and other internal/external forces. The GDE is utilized in discrete form as a population balance on each cluster or particle size. The methodology uses the nodal/sectional method of to approximate the GDE [23-26]. This approach effectively divides the aerosol population into three classes, monomers, clusters, and particles [16]. The GDE is therefore solved as a set of $N_{s}$ transport equations, one for each bin $Q_{k}$, $k=1,2, \ldots, N_{s}$ [27]. Monomers of size $0.5 \mathrm{~nm}$ in diameter populate bin 1 while bins 2 and 3 are populated by clusters of molecules. Molecular clusters of size $1 \mathrm{~nm}$ and larger are considered "particles" [28]. The general transport equation for the concentration of monomers, clusters, and particles in bin $k, Q_{k}$, is written as

$$
\frac{\partial \rho Q_{k}}{\partial t}+\frac{\partial \rho u_{j} Q_{k}}{\partial x_{j}}=\frac{\partial}{\partial x_{j}}\left(\rho D_{Q} \frac{\partial Q_{k}}{\partial x_{j}}\right)+\dot{\omega}_{k}^{Q},
$$

where $D_{Q}$ is the diffusivity given by

$$
D_{\mathrm{Q}}=k_{b} T \frac{C_{c}}{3 \pi \mu d_{p}},
$$

where $k_{b}$ is the Boltzmann constant, $C_{c}$ is the Cunningham correction factor, and $d_{p}$ is the mean volume particle diameter [29]. The source term $\dot{\omega}_{k}^{Q}$ represents particle formation and growth processes and is given by

$$
\dot{\omega}_{k}^{Q}= \begin{cases}J-\rho \sum_{i=1}^{N_{s}} \beta_{i 1} Q_{i} Q_{1}, & k=1, \\ \frac{1}{2} \sum_{i=1}^{N_{s}} \sum_{j=1}^{N_{s}} \chi_{i j k} \rho \beta_{i j} Q_{i} Q_{j}-\rho \sum_{i=1}^{N_{s}} \beta_{i k} Q_{i} Q_{k}, & k>1,\end{cases}
$$

where $J$ accounts for the formation of monomers in bin $k=1$ via chemical reaction [30-33] and $\chi_{i j k}$ is given by

$$
\chi_{i j k}= \begin{cases}\frac{v_{k+1}-\left(v_{i}+v_{j}\right)}{v_{k+1}-v_{k}}, & \text { if } v_{k} \leq v_{i}+v_{j}<v_{k+1}, \\ \frac{\left(v_{i}+v_{j}\right)-v_{k-1}}{v_{k}-v_{k-1}}, & \text { if } v_{k-1} \leq v_{i}+v_{j}<v_{k}, \\ 0, & \text { otherwise. }\end{cases}
$$


The source term in (6), $\omega_{k}^{Q}$, represents the effects of nucleation, condensation (via monomer-cluster, monomerparticle and cluster-particle collisions), and Brownian coagulation. In this study, all particle formation and growth processes occur at $300 \mathrm{~K}$. This means that all particles considered are agglomerates, meaning growth via coagulation results in fractal-like aggregate particles, consisting of primary particles (monomers). The collision frequency function, $\beta_{i j}$, is well documented in both the free-molecular and continuum regimes [29, 34-37]. Collisions of all particles (monomers, clusters, etc.) are considered and $\beta_{i j}$ is given by

$$
\beta_{i j}= \begin{cases}a_{1}\left(i^{1 / D_{f}}+j^{1 / D_{f}}\right)^{D_{f}}\left(\frac{1}{i}+\frac{1}{j}\right)^{1 / 2}, & D_{f}<2 \text { free-molecular regime, } \\ a\left(i^{1 / D_{f}}+j^{1 / D_{f}}\right)^{2}\left(\frac{1}{i}+\frac{1}{j}\right)^{1 / 2}, & D_{f} \geq 2 \text { free-molecular regime, } \\ \frac{2 k_{b} T}{3 \mu}\left(\frac{1}{v_{i}^{1 / D_{f}}}+\frac{1}{v_{j}^{1 / D_{f}}}\right)\left(v_{i}^{1 / D_{f}}+v_{j}^{1 / D_{f}}\right), & \text { in continuum regime, }\end{cases}
$$

where

$$
\begin{aligned}
& a=\left(\frac{3 v_{o}}{4 \pi}\right)^{1 / 6}\left(\frac{6 k_{b} T}{\rho_{p}}\right)^{1 / 2}, \\
& a_{1}=\frac{2^{D_{f}} a}{4.89},
\end{aligned}
$$

where $v_{o}$ is the primary particle volume, $v_{i}$ is the volume of an agglomerate in the $i$ th section, $\rho_{p}$ is the particle density, $i$ and $j$ are the primary particle numbers in bins $i$ and $j$, respectively, and $D_{f}$ is the fractal dimension. The particles generated by the industrial aerosol processes are usually nonspherical and the products are composed of groups of adhering particles ranging from loosely linked particles, sometimes termed agglomerates, to strongly necked particles, called aggregates or hard agglomerates. The fractal dimension is used to represent the shape of the aggregate or particle structure. A statistical concept, the fractal (or Hausdorff) dimension $D_{f}$, was introduced to describe the shape of the agglomerate, which is obtained after averaging over many agglomerates with the same number of primary particles. The value of the fractal dimension ranges from $D_{f}=1$ to $D_{f}=$ 3 depending on the details of the agglomerate formation process [29]. The bins are organized in such a manner that the volume of particles in two successive bins is doubled; that is, $v_{k}=2 \times v_{k-1}[23,38]$.

\section{Results}

3.1. Flow Configuration. The flow under consideration is a three-dimensional, isothermal, turbulent reacting jet issuing from an orifice of diameter $D$ into a coflowing stream. The jet is composed of titanium tetrachloride $\left(\mathrm{TiCl}_{4}\right)$ diluted in nitrogen $\left(\mathrm{N}_{2}\right)$, while the coflowing stream is composed of water vapor $\left(\mathrm{H}_{2} \mathrm{O}\right)$ diluted in $\mathrm{N}_{2}$. The initial velocities are $U_{o}=100 \mathrm{~m} / \mathrm{s}$ for the jet and $U_{\infty}$ for the coflowing stream. The fluid field is characterized by the velocity ratio $U_{\infty} / U_{o}=0.2$. The Reynolds number based on velocity of the high-speed stream and the jet diameter is $\operatorname{Re}=U_{o} D / \nu=3,000$. The simulation is performed at $300 \mathrm{~K}$ and atmosphere pressure ( $1 \mathrm{~atm})$. To accelerate the development of large-scale structures, random perturbations with a maximum intensity of $5 \%$ are added to the cross-stream $v$-velocity. The chemical composition of the jet stream is $0.01 \% \mathrm{TiCl}_{4}$ and $99.99 \% \mathrm{~N}_{2}$ by mass. The simulation utilizes stoichiometric mixtures and the molar ratio of $1: 2$ for $\mathrm{TiCl}_{4}$ and $\mathrm{H}_{2} \mathrm{O}$.

In this work, several assumptions and approximations are utilized. These are stated below for clarity.

(1) Ceramic powders such as $\mathrm{TiO}_{2}$ have low equilibrium vapor pressures implying that single molecules may be considered particles [31]. As $\mathrm{TiO}_{2}$ is formed, it appears as $0.5 \mathrm{~nm}$ diameter free spherical $\mathrm{TiO}_{2}$ "monomers," populating bin $k=1$; hence nucleation is treated as an instantaneous process.

(2) The nanoparticles are small enough to follow the fluid path lines. Additionally, the particle volume fraction is of order $10^{-7}$. As a result the presence of the particles does not affect the fluid field.

(3) Condensation is dominated by the collision rate of monomers with other monomers, clusters, and particles. This means the condensable species in the simulations is the $\mathrm{TiO}_{2}$ "vapor."

(4) The clusters and particles are stable because of the high supersaturation of the monomers. As a result, at these temperatures, there is no evaporation or sublimation of particles back to the gas-phase.

(5) The fractal dimension, $D_{f}$, is based on the collision time, $\tau_{c}$, and sintering time, $\tau_{f}$. In this work, all processes occur at $T=300 \mathrm{~K}$. At this temperature, $\mathrm{TiO}_{2}$ particles do not sinter as $\tau_{c} \ll \tau_{f}$ [39].

(6) A fractal dimension of $D_{f}=3$ is used when collisions occur between monomers while $D_{f}=2$ is used when monomers and dimers collide. For all other particle interactions (collisions between larger particles) a fractal dimension of $D_{f}=1.8$ is used $[39,40]$. 
3.2. Numerical Specifications. Ten bins are used to discretize the particle field $\left(N_{s}=10\right)$. The computational domain is of size $20 D \times 15 D \times 4 D$ and is comprised of $500 \times 375 \times 100$ grid points in the $x$-, $y$-, and $z$-directions, respectively. The governing transport equations representing both the fluid and particle fields are solved using a MacCormack-based finite difference scheme $[41,42]$. The scheme is of second order, accurate in time, and of fourth order, accurate in space. The boundary conditions are periodic in the spanwise $z$-direction and zero-derivative in the cross-stream $y$ direction and nonreflecting boundary conditions are used in both inflow and outflow boundaries ( $x$-direction) [43]. The simulation is performed up to a nondimensional time of $t^{\star}=U_{o} t / D=100$ which corresponds to a physical time of $0.48 \mathrm{~ms}$. Both instantaneous and mean or averaged data are presented. We average in the $z$-direction as it is the spatially homogeneous direction in planar jets. Quantities such as contours, isosurfaces, and mean data are useful in making qualitative and quantitative assessments of the nanoparticle growth dynamics as well as the underlying fluid and particle fields.

3.3. Flow Field. The vorticity is the curl of the velocity vector and is an indicator of fluid mixing. The vorticity magnitude is the local rate of rotation. In nonpremixed chemically reacting flows, vorticity has the effect of increasing the interfacial area between the reactants. An isosurface of the instantaneous vorticity magnitude, the $|\omega|=3$ level-set, is shown in Figure 1 at time $t^{\star}=100$. The image shows that the flow is initially laminar and becomes turbulent as the jet travels downstream. Near $x / D=6$, the two boundary layers initially located at $y / D= \pm 0.5$ merge and the jet spreads across the domain. This is aided by the presence of vortex braids, the tubular structures oriented in the stream-wise $x$-direction, which act to draw the surrounding fluid into contact with the fluid issuing through the nozzle. Further downstream, vortex bending and stretching acts to generate small-scale structures as the flow becomes fully turbulent. It is evident that the jet suddenly spreads/amplifies near $x / D=8$. Near $x / D=10$, the contours reveal a high concentration of intense mixing that persists throughout the latter half of the computational domain. These small-scale structures result in an increased chemical reaction as they serve to bring the reactants into contact. High-resolution DNS facilitates the capturing of the small-scale structures. If turbulence models were used, then the effects of the small-scale interactions on the chemical reaction (and particle formation and growth) would need to be accounted for $[18,44]$.

3.4. Titanium Dioxide. The chemical conversion of the $\mathrm{TiCl}_{4}$ and $\mathrm{H}_{2} \mathrm{O}$ to produce $\mathrm{TiO}_{2}$ is the first step in the particle synthesis process. Instantaneous contours of the $\mathrm{TiO}_{2}$ mass fraction at the $z=0$ plane and time $t^{\star}=100$ are shown in Figure 2. The image reveals that $\mathrm{TiO}_{2}$ is initially formed along the interface of the two streams and subsequently where the reactants are well mixed. The $\mathrm{TiO}_{2}$ is convected downstream and across the jet. The maximum $\mathrm{TiO}_{2}$ mass fraction appears far downstream and $\mathrm{TiO}_{2}$ mass spreads out. Because of

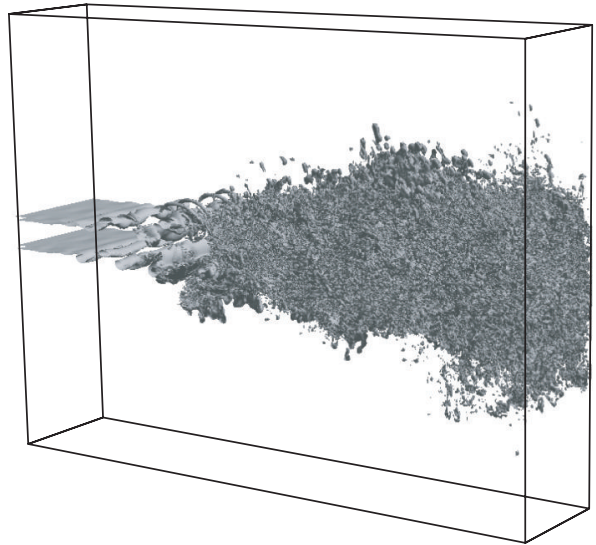

FIGURE 1: An instantaneous isosurface of the vorticity magnitude, $|\omega|=3$, at time $t^{\star}=100$.

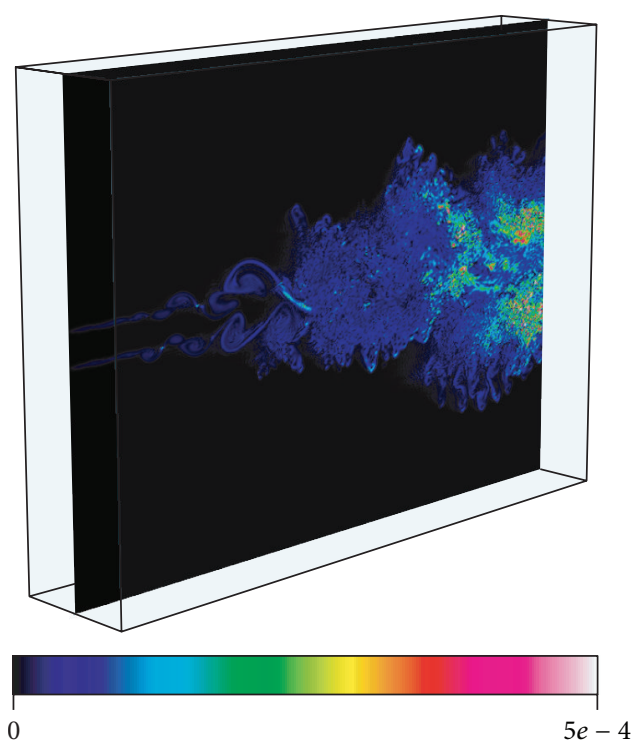

FIGURE 2: Instantaneous contour of the $\mathrm{TiO}_{2}$ mass fraction at $z=0$ plane at $t^{\star}=100$.

the infinite-rate chemistry, at least one of the reactants is consumed immediately upon contact. Turbulent mixing brings "fresh" reactants together (via large-scale transport) to produce $\mathrm{TiO}_{2}$ (via molecular scale transport). As the $\mathrm{TiO}_{2}$ is produced, molecular diffusion acts to transport it from $\mathrm{TiO}_{2}$ rich to $\mathrm{TiO}_{2}$-free regions.

3.5. Particle Concentrations. As the chemical reaction proceeds, more titania is produced. The monomers collide with each other to produce dimers; those dimers collide with monomers (condensation) to produce trimers and collide with each other to produce larger particles (coagulation). An advantage of the nodal approach is the fact that the particle field is obtained as a function of size (in addition to space and time). A detailed view of the $\mathrm{TiO}_{2}$ nanoparticle field can be obtained by observing the particle number concentrations distributed throughout the domain. Instantaneous contours 

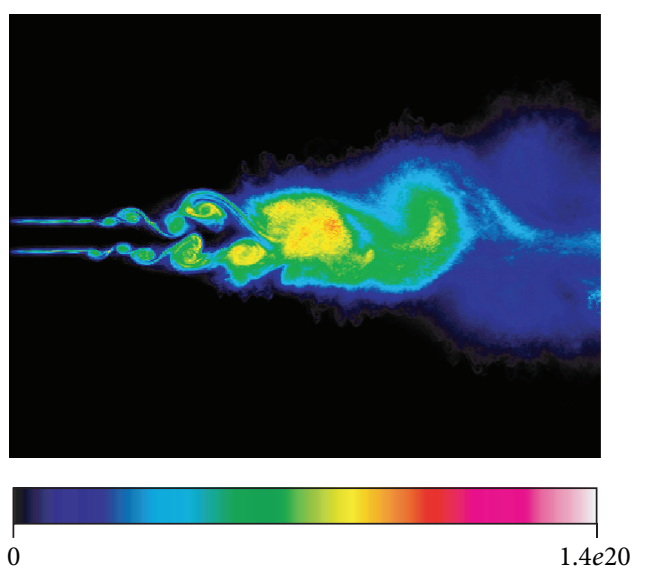

(a)
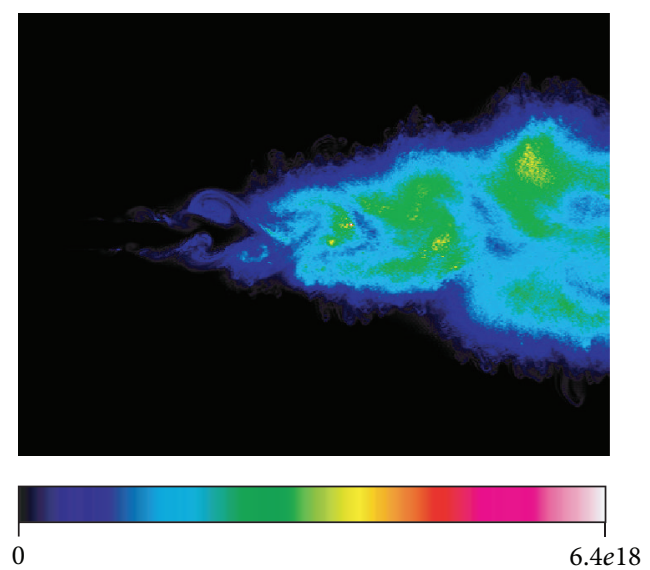

(b)

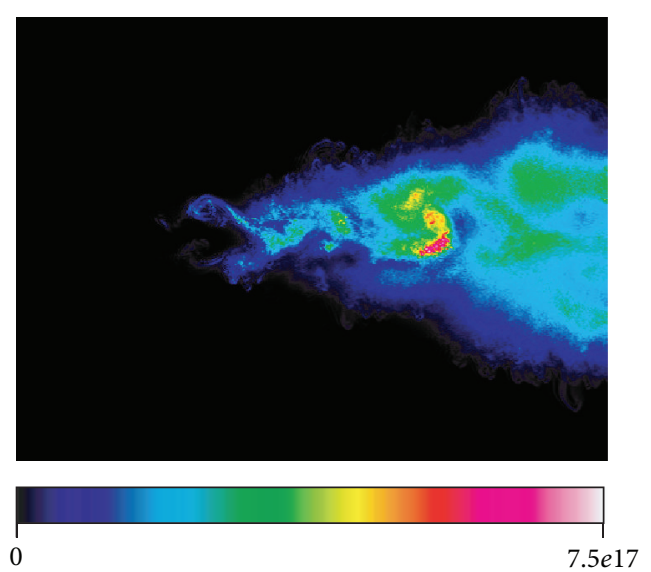

(c)

FIGURE 3: Instantaneous contours of the $z$-direction averaged particle number concentrations: (a) monomers; (b) $1 \mathrm{~nm}$; (c) $2 \mathrm{~nm}$.

of the $z$-direction averaged particle number concentrations of monomers, $1 \mathrm{~nm}$ and $2 \mathrm{~nm}$ particles at $t^{\star}=100$, are shown in Figure 3. Figure 3(a) shows that monomers initially appear at the interface of the two streams and the concentration increases significantly near $x / D=10$, after collapse of the jet potential core. Figure 3(b) shows that the $1 \mathrm{~nm}$ diameter particles begin to appear near $x / D=5$, and high concentrations are found between $x / D=10$ and $x / D=$ 20. Figure 3(c) shows a similar trend for the $2 \mathrm{~nm}$ particles.

To convey the spatial inhomogeneity of the particle field, a three-dimensional view is presented in Figure 4. The figure shows three isosurfaces colored to show the large concentration of $1 \mathrm{~nm}, 2 \mathrm{~nm}$, and $3 \mathrm{~nm}$ nanoparticles. The presence of $1 \mathrm{~nm}$ particles (colored green) throughout the domain reflects the ongoing chemical reaction and nucleation that occurs when large-scale convective mixing brings $\mathrm{TiCl}_{4}$ and $\mathrm{H}_{2} \mathrm{O}$ into contact. The increase in particle size with downstream distance is evident in the image as the $3 \mathrm{~nm}$ diameter particles are only found in the last third of the domain.

3.6. Mean Nanoparticle Size and Geometric Standard Deviation. Particle size distributions (PSDs) are often characterized by the mean diameter and the geometric standard deviation (GSD). Though the nodal approach employed contains the full PSD, conveying that all of the information is not trivial $[45,46]$, the information conveyed by the first two moments can be quite useful. The mean diameter used here is the volume-equivalent mean particle diameter and is given by $d_{p}=\left(6 v_{p} / \pi\right)^{1 / 3}$, where the mean volume is given by

$$
v_{p}=\frac{\sum_{i=1}^{N_{s}} Q_{i} v_{i}}{\sum_{i=1}^{N_{s}} Q_{i}} .
$$

The GSD represents the width of the PSD and is given by

$$
\left[\log \left(\sigma_{g}\right)\right]^{2}=\frac{\sum_{k=1}^{N_{s}} Q_{k}\left(\log \left(d_{p_{k}}\right)-\log \left(\tilde{d}_{p_{k}}\right)\right)^{2}}{\sum_{k=1}^{N_{s}} Q_{k}}
$$

where $\widetilde{d}_{p_{k}}$ is a number mean diameter given by

$$
\log \left(\tilde{d}_{p_{k}}\right)=\frac{\sum_{k=1}^{N_{s}} Q_{k} \log \left(d_{p_{k}}\right)}{\sum_{k=1}^{N_{s}} Q_{k}}
$$

Larger GSD values indicate that the size distribution is relatively broad while smaller GSD values $\left(\sigma_{g}=1\right)$ indicate a relatively narrow distribution [47]. The GSD for the 


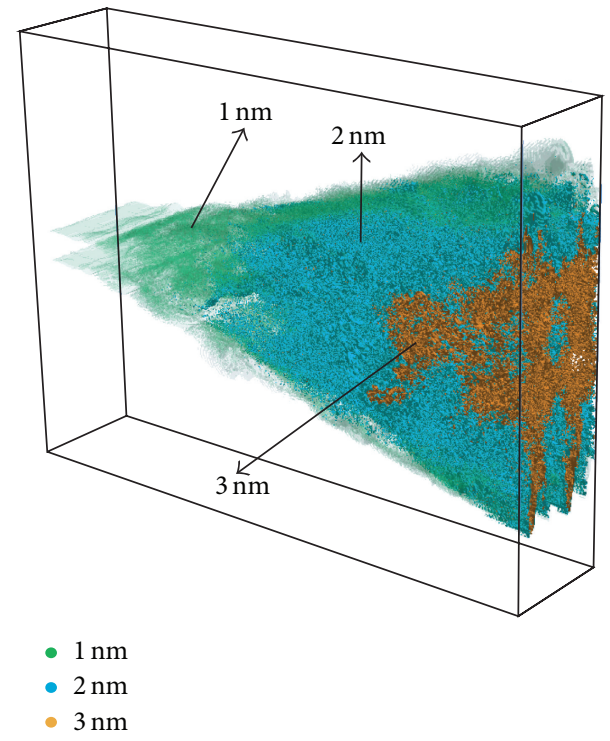

FIGURE 4: Instantaneous isosurfaces of $1 \mathrm{~nm}, 2 \mathrm{~nm}$, and $3 \mathrm{~nm}$ particles at $t^{\star}=100$.

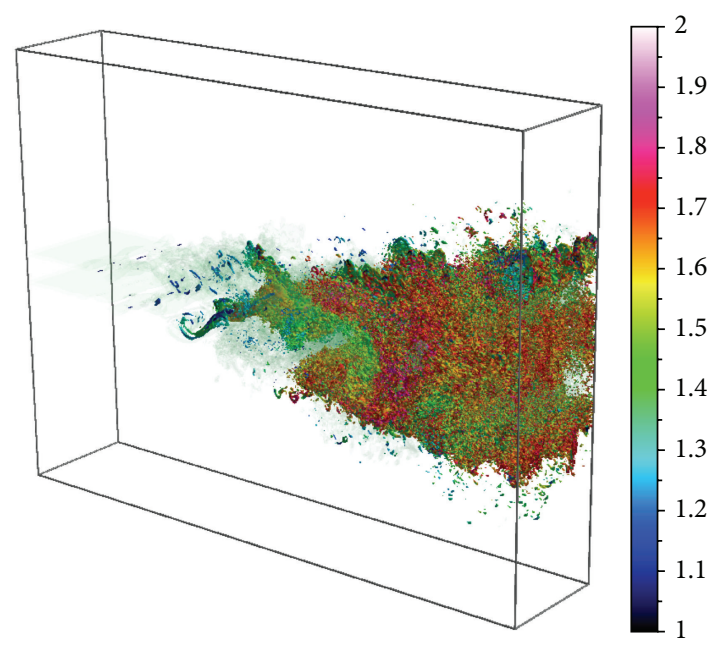

FIGURE 5: An instantaneous isosurface of vorticity colored by the geometric standard deviation, $\sigma_{g}$.

hydrolysis of $\mathrm{TiCl}_{4}$ in a planar jet is shown on an isosurface of vorticity magnitude at time $t^{\star}=100$ in Figure 5 . The figure reveals that the GSD generally increases as the jet travels downstream. This implies that mixing (due to turbulence) is a significant contribution to particle polydispersity. If nucleation were absent, there would be no monomers, and the coagulating particles would achieve the so-called selfpreserving value $\left(\sigma_{g}=1.5\right)[48,49]$. Locations where $\sigma_{g}>$ 1.5, in Figure 5, reflect where nucleation, condensation, and coagulation simultaneously occur. That is, "newly" formed particles by nucleation/mixing and old particles generated by coagulation exist.

More insight into the particle field may be obtained by considering the relationship between the mean diameter and the GSD. A scatter plot of $\sigma_{g}$ versus $d_{p}$ is shown in Figure 6.

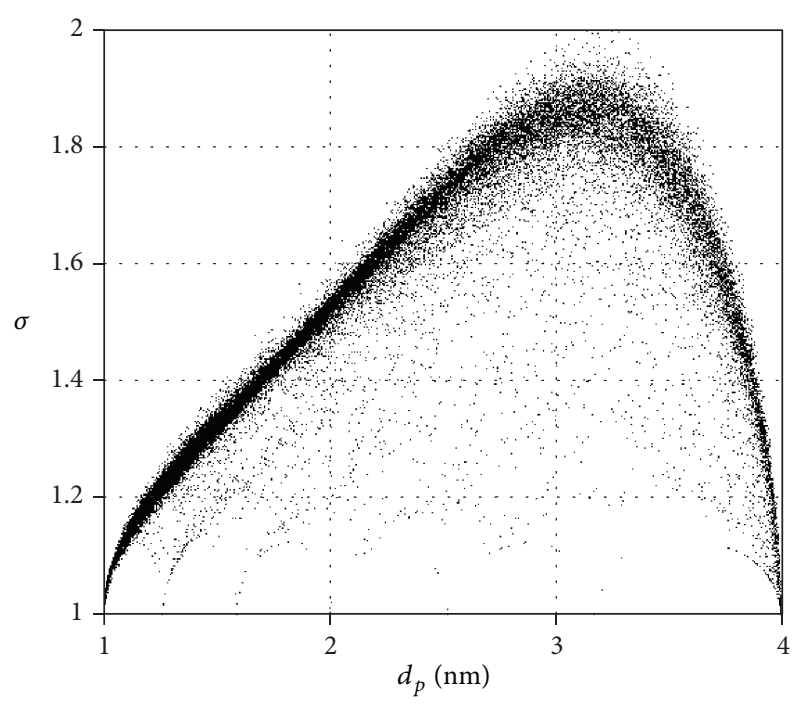

FIGURE 6: Scatter plot of the geometric standard deviation, $\sigma_{g}$, versus the mean particle diameter, $d_{p}$.

The figure shows that at the two ends of the size distribution, $d_{p}=1 \mathrm{~nm}$ and $d_{p}=4 \mathrm{~nm}$, the distribution is fairly narrow or unimodal, while the GSD is largest $\left(1.8<\sigma_{g}<2\right)$ when the mean diameter is near $d_{p}=3.2 \mathrm{~nm}$. This relatively large GSD indicates that there are a variety of particle dynamics present in regions of the flow where the mean diameter is $d_{p}=3.2 \mathrm{~nm}$.

3.7. Particle Growth. The particle growth-rate is an important parameter to consider as, in combination with residence time or reactor size, it is a predictor of particle size. A diameterbased growth-rate $\Omega_{\mathrm{nm}}$ (with units of $\mathrm{nm} / \mathrm{s}$ ) is defined based on

$$
\Omega_{\mathrm{nm}}=\left(\frac{6 \Omega}{\pi}\right)^{1 / 3} \times 10^{9},
$$

where $\Omega$ is the particle volumetric growth-rate given by

$$
\Omega=\frac{\sum_{k}^{N_{s}}\left(v_{k}\right)^{2} \omega_{k}}{\sum_{k}^{N_{s}} Q_{k} v_{k}}-\frac{\sum_{k}^{N_{s}}\left(v_{k}\right)^{2} Q_{k}}{\left(\sum_{k}^{N_{s}} Q_{k} v_{k}\right)^{2}} \sum_{k}^{N_{s}} v_{k} \omega_{k} .
$$

A contour plot of the $z$-averaged growth-rate, $\left\langle\Omega_{\mathrm{nm}}\right\rangle_{z}$, is shown in Figure 7. The figure shows that within the first 6 diameters, $0 \leq x / D \leq 6$, growth is confined to roughly $\left\langle\Omega_{\mathrm{nm}}\right\rangle_{z}=10 \mathrm{~nm} / \mathrm{s}$ at the interface of the jet and the coflowing stream, where the hydrolysis reaction produces $\mathrm{TiO}_{2}$ vapor. Near $x / D=10$, when the shear layers merge, the particles begin to grow faster, with the rate approaching $\left\langle\Omega_{\mathrm{nm}}\right\rangle_{z}=25 \mathrm{~nm} / \mathrm{s}$. This occurs after the potential core collapses. Further downstream, the growth-rate increases to roughly $\left\langle\Omega_{\mathrm{nm}}\right\rangle_{z}=50 \mathrm{~nm} / \mathrm{s}$ and this value is maintained for $x / D>14$. (It should be noted that this is the average growthrate and at other points in the domain the values of $\Omega_{\mathrm{nm}}$ may be smaller or larger.)

The particles grow via two mechanisms: condensation (collisions between monomers and particles) and 


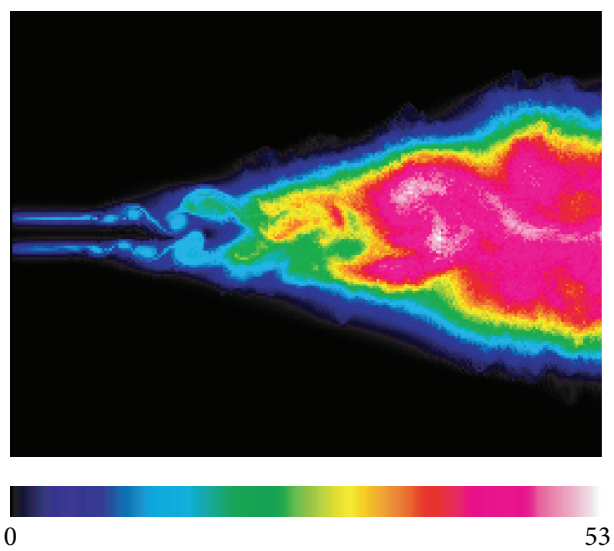

FIGURE 7: Instantaneous contours of the $z$-direction averaged particle growth-rate, $\left\langle\Omega_{\mathrm{nm}}\right\rangle_{z}$ at $t^{\star}=100$.

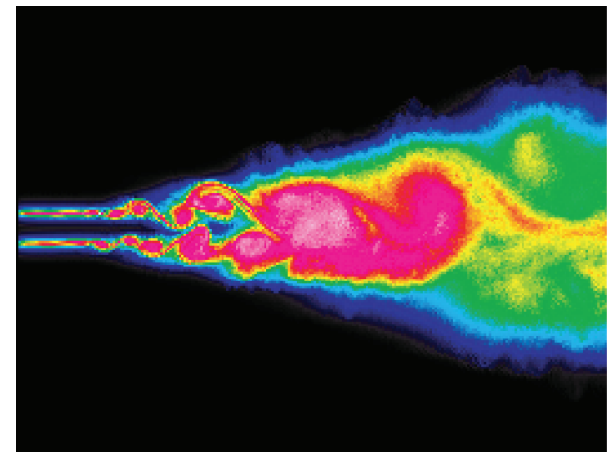

0

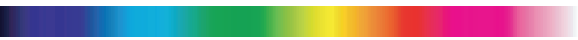

28

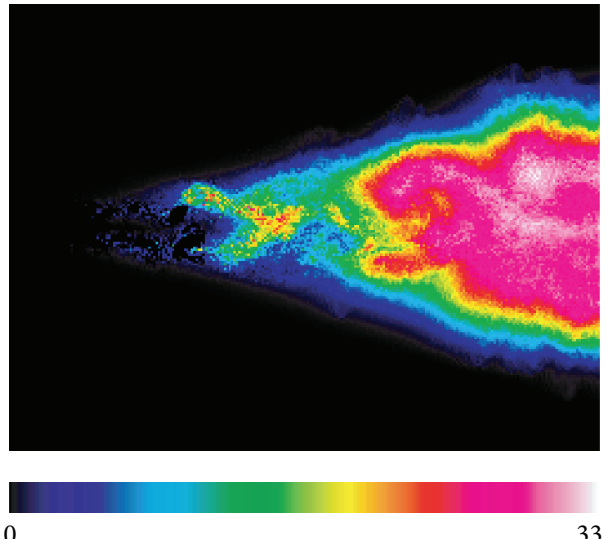

(b)

FIGURE 8: Instantaneous contours of the nanoparticle growth-rate decomposed by mechanism; $z$-direction averaged particle growth-rate (a) by condensation, (b) by coagulation.

coagulation (collisions between particles). Because the particle data is available as a function of size, the contribution of each mechanism is readily available. Particle growth, by mechanism, is shown in Figure 8. The interactions between monomers and particles, or the condensation growth-rate, is shown in Figure 8(a). (This image looks similar to the monomer number concentration shown in Figure 3(a) because the condensation is represented by the collision between monomers and particles.) The contours show that large condensation growth occurs both in the proximal region of the jet and after collapse of the jet core. This reflects the ongoing hydrolysis of $\mathrm{TiCl}_{4}$ and production of $\mathrm{TiO}_{2}$ and its deposition on existing particles.

Particle growth by coagulation is shown in Figure 8(b). The contours of the $z$-averaged growth-rate shows that growth by coagulation begins after collapse of the jet potential core. The growth-rate in the region $4<x / D<8$ is as high as $\Omega_{\mathrm{nm}}=16 \mathrm{~nm} / \mathrm{s}$. Farther downstream the $z$-averaged coagulation growth-rate doubles. This region of the flow is dominated by mixing and small-scale turbulence (evident in Figure 1). That region of the flow also contains particles of a variety of sizes, small and large, as reflected by $\sigma_{g}$ in Figure 5. The small-scale turbulence means dissipation and increased residence times, while the disparate particle sizes mean an efficient collision efficiency. These two combine to increase coagulation.

The spatial relationship between condensation growth and coagulation growth is elucidated by showing the contribution of each at every grid point in the computational domain. A scatter plot of the two growth-rates is shown in Figure 9. The growth-rates are not averaged in the $z$-direction and while a spatial relationship is not directly evident from this figure, one may be reliably inferred along with the previous data. Figure 9 shows that where condensation growth is low, coagulation growth is high. This trend is evident in Figure 8 as well. However, Figure 9 shows that in these regions the coagulation growth-rate can be as much as an 


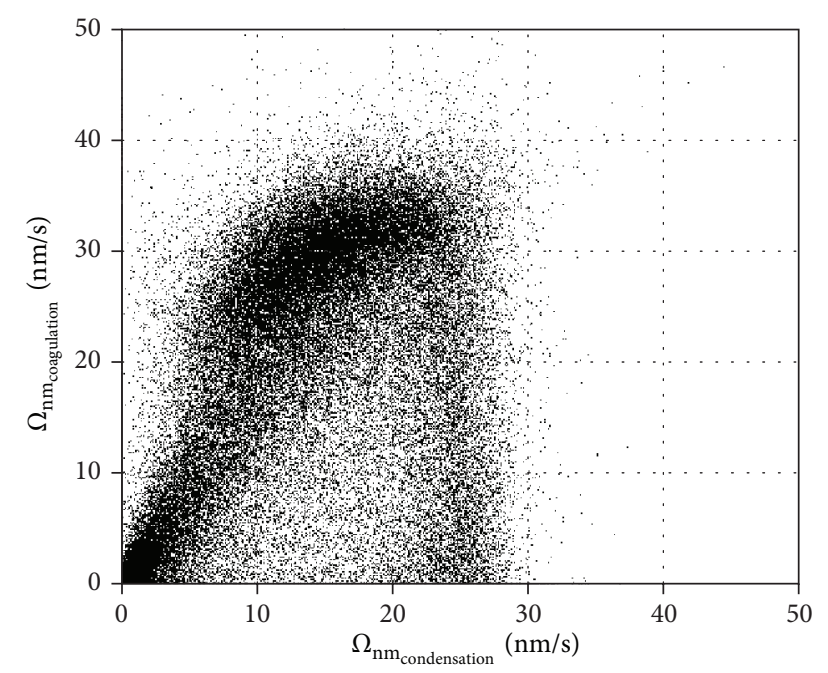

FIGURE 9: Scatter plot of the particle growth-rate by condensation versus the particle growth-rate by coagulation.

order of magnitude greater than the condensation growthrate.

\section{Summary and Conclusions}

The growth mechanisms of titania during hydrolysis of titanium tetrachloride in a three-dimensional planar jet are studied via direct numerical simulation. The mass, momentum, enthalpy, and species transport equations are solved in a model-free manner. Titania was produced via the hydrolysis of titanium tetrachloride, modeled via a 1-step infinitely fast chemical mechanism. The particle field was represented using a nodal method and solves for the evolution of the concentration of particles of various sizes in an Eulerian manner. When coupled to the Navier-Stokes solver, the fluid, thermal, chemical, and particle fields are obtained as a function of space and time.

The results show that fluid turbulence/gas mixing plays a very important role in particle growth. The results indicate that the particle formation and growth are greatly affected if not dominated by mixing and chemical reaction. Reactant conversion or titania production is limited by the ability of the flow to bring the $\mathrm{TiCl}_{4}$ and $\mathrm{H}_{2} \mathrm{O}$ into contact. Evidence for this is the particle formation throughout the domain. Additionally, the results show that where the turbulence is more intense, the particles are larger and the particle size distribution, as characterized by the geometric standard deviation, is wider. Particle growth in the proximal region of the jet is dominated by condensation. After collapse of the jet core, nanoparticle growth due to coagulation increases significantly. Though $\mathrm{TiO}_{2}$ is produced after core collapse, producing condensible species, particle growth due to coagulation is as much as an order of magnitude greater than that due to condensation. While both condensation and coagulation act to increase polydispersity, coagulation has been shown to increase the width of the particle size distribution faster, or greater, than condensation [50].
These results help to shed light on, and improve our understanding of, the underlying growth dynamics occurring in nanoparticle synthesis processes. The change from condensation-dominated to coagulation-dominated growth is useful in modeling the complete synthesis process, including sintering, and the formation of hard and soft agglomerates. Spanning the size range from single molecules (particle inception) to hundreds of nanometers, as the particles found in many industrial processes and applications, is compute intensive [18]. However, knowing that condensation and molecular growth is relatively minor could facilitate the use of more affordable modeling strategies that do not need to account for every phenomena [6,51]. Additionally, a highly desired attribute of metal oxide nanoparticles is their specific surface area. The ability to synthesize narrow size distributions which are desired is advantageous in that it removes the processing necessary to separate particles by size. This is advantageous in that it lowers cost [52-55].

One strategy to reduce polydispersity may be to delay the transition to turbulence vis a vis delaying collapse of the jet core. While the particle field is known as a function of size, it should be noted that in this work we are unable to distinguish the various phases of titania, rutile, anatase, or brookite [56, 57]. The phase composition of titania is very much a function of the synthesis process, for example, precursor composition and temperature history, which are greatly simplified in this work. Such capability requires phenomenological models that account for particle dynamics as a function of space, time, and temperature, as well as solid-state diffusion processes, and is quite beyond the scope of this work.

\section{Conflict of Interests}

The author declares that there is no conflict of interests regarding the publication of this paper.

\section{Acknowledgments}

Funding for this research was provided by the University of Minnesota. Computational resources were provided by Minnesota Supercomputing Institute.

\section{References}

[1] S. E. Pratsinis and S. Vemury, "Particle formation in gases: a review," Powder Technology, vol. 88, no. 3, pp. 267-273, 1996.

[2] G. Beaucage, H. Kammler, P. Mueller et al., "Probing the dynamics of nanoparticle growth in a flame using synchrotron radiation," Nature Materials, vol. 3, no. 6, pp. 370-373, 2004.

[3] S. Panda and S. E. Pratsinis, "Modeling the synthesis of aluminum particles by evaporation-condensation in an aerosol flow reactor," Nanostructured Materials, vol. 5, no. 7-8, pp. 755767, 1995.

[4] S. Yuu, K. Ikeda, and T. Umekage, "Flow-field prediction and experimental verification of low Reynolds number gas-particle turbulent jets," Colloids and Surfaces A: Physicochemical and Engineering Aspects, vol. 109, pp. 13-27, 1996.

[5] S. Tsantilis, H. K. Kammler, and S. E. Pratsinis, "Population balance modeling of flame synthesis of titania nanoparticles," 
Chemical Engineering Science, vol. 57, no. 12, pp. 2139-2156, 2002.

[6] N. Settumba and S. C. Garrick, "Direct numerical simulation of nanoparticle coagulation in a temporal mixing layer via a moment method," Journal of Aerosol Science, vol. 34, no. 2, pp. 149-167, 2003.

[7] D. L. Marchisio and R. O. Fox, "Solution of population balance equations using the direct quadrature mehtod of moments," Journal of Aerosol Science, vol. 36, pp. 43-73, 2005.

[8] F. Aristizabal, R. J. Munz, and D. Berk, "Modeling of the production of ultra fine Aluminium particles in rapid quenching turbulent flow," Journal of Aerosol Science, vol. 37, no. 2, pp. 162186, 2006.

[9] S. Rigopoulos, "PDF method for population balance in turbulent reactive flow," Chemical Engineering Science, vol. 62, no. 23, pp. 6865-6878, 2007.

[10] K. Zhou, A. Attili, A. Alshaarawi, and F. Bisetti, "Simulation of aerosol nucleation and growth in a turbulent mixing layer," Physics of Fluids, vol. 26, no. 6, Article ID 065106, 2014.

[11] S. A. Orszag and I. Staroselsky, "CFD: progress and problems," Computer Physics Communications, vol. 127, no. 1, pp. 165-171, 2000.

[12] K. Nakaso, T. Fujimoto, T. Seto, M. Shimada, K. Okuyama, and M. M. Lunden, "Size distribution change of titania nanoparticle agglomerates generated by gas phase reaction, agglomeration, and sintering," Aerosol Science and Technology, vol. 35, no. 5, pp. 929-947, 2001.

[13] T. Johannessen, S. E. Pratsinis, and H. Livbjerg, "Computational analysis of coagulation and coalescence in the flame synthesis of titania particles," Powder Technology, vol. 118, no. 3, pp. 242-250, 2001.

[14] E. G. Moody and L. R. Collins, "Effect of mixing on the nucleation and growth of titania particles," Aerosol Science and Technology, vol. 37, no. 5, pp. 403-424, 2003.

[15] G. Wang and S. C. Garrick, "Modeling and simulation of titania formation and growth in temporal mixing layers," Journal of Aerosol Science, vol. 37, no. 4, pp. 431-451, 2006.

[16] S. Das and S. C. Garrick, "The effects of turbulence on nanoparticle growth in turbulent reacting jets," Physics of Fluids, vol. 22, no. 10, Article ID 103303, 2010.

[17] S. C. Garrick, "Effects of turbulent fluctuations on nanoparticle coagulation in shear flows," Aerosol Science and Technology, vol. 45, no. 10, pp. 1272-1285, 2011.

[18] A. J. Fager, J. Liu, and S. C. Garrick, "Hybrid simulations of metal particle nucleation: a priori and a posteriori analyses of the effects of unresolved scalar interactions on nanoparticle nucleation," Physics of Fluids, vol. 24, no. 7, Article ID 075110, 2012.

[19] N. J. Murfield and S. C. Garrick, "Large eddy simulation and direct numerical simulation of homogeneous nucleation in turbulent wakes," Journal of Aerosol Science, vol. 60, pp. 21-33, 2013.

[20] N. J. Murfield and S. C. Garrick, "The effects of unresolved scalar fluctuations during homogeneous nucleation," Aerosol Science and Technology, vol. 47, no. 7, pp. 806-817, 2013.

[21] P. Givi, "Model free simulations of turbulent reactive flows," Progress in Energy and Combustion Science, vol. 15, no. 1, pp. 1107, 1989.

[22] K. E. J. Lehtinen and M. R. Zachariah, "Self-preserving theory for the volume distribution of particles undergoing brownian coagulation," Journal of Colloid and Interface Science, vol. 242, no. 2, pp. 314-318, 2001.
[23] S. C. Garrick, K. E. J. Lehtinen, and M. R. Zachariah, "Nanoparticle coagulation via a Navier-Stokes/nodal methodology: evolution of the particle field," Journal of Aerosol Science, vol. 37, no. 5, pp. 555-576, 2006.

[24] F. Gelbard and J. H. Seinfeld, "Simulation of multicomponent aerosol dynamics," Journal of Colloid And Interface Science, vol. 78, no. 2, pp. 485-501, 1980.

[25] P. Biswas, C. Y. Wu, M. R. Zachariah, and B. McMillin, "Characterization of iron oxide-silica nanocomposites in flames: part II: comparison of discrete-sectional model predictions to experimental data," Journal of Materials Research, vol. 12, no. 3, pp. 714-723, 1997.

[26] K. E. J. Lehtinen and M. R. Zachariah, "Energy accumulation in nanoparticle collision and coalescence processes," Journal of Aerosol Science, vol. 33, no. 2, pp. 357-368, 2002.

[27] G. Wang and S. C. Garrick, "Modeling and simulation of titania formation and growth in temporal mixing layers," Journal of Aerosol Science, vol. 37, no. 4, pp. 431-451, 2006.

[28] J. Loeffler, S. Das, and S. C. Garrick, "Large eddy simulation of titanium dioxide nanoparticle formation and growth in turbulent jets," Aerosol Science and Technology, vol. 45, no. 5, pp. 616-628, 2011.

[29] K. S. Friedlander, Smoke, Dust and Haze: Fundamentals of Aerosol Dynamics, Oxford University Press, New York, NY, USA, 2000.

[30] M. Frenklach and S. J. Harris, "Aerosol dynamics modeling using the method of moments," Journal of Colloid and Interface Science, vol. 118, no. 1, pp. 252-261, 1987.

[31] S. E. Pratsinis, "Particle production by gas-to-particle conversion in turbulent flows," Journal of Aerosol Science, vol. 20, no. 8, pp. 1461-1464, 1989.

[32] J. D. Landgrebe and S. E. Pratsinis, "A discrete-sectional model for particulate production by gas-phase chemical reaction and aerosol coagulation in the free-molecular regime," Journal of Colloid and Interface Science, vol. 139, no. 1, pp. 63-86, 1990.

[33] G. Wang and S. C. Garrick, "Modeling and simulation of titania synthesis in two-dimensional methane-air flames," Journal of Nanoparticle Research, vol. 7, no. 6, pp. 621-632, 2005.

[34] G. W. Mulholland, R. J. Samson, R. D. Mountain, and M. H. Ernst, "Cluster size distribution for free molecular agglomeration," Energy \& Fuels, vol. 2, no. 4, pp. 481-486, 1988.

[35] J. Cai, N. Lu, and C. M. Sorensen, "Analysis of fractal cluster morphology parameters: structural coefficient and density autocorrelation function cutoff," Journal of Colloid And Interface Science, vol. 171, no. 2, pp. 470-473, 1995.

[36] R. Jullien and P. Meakin, "Simple models for the restructuring of three-dimensional ballistic aggregates," Journal of Colloid And Interface Science, vol. 127, no. 1, pp. 265-272, 1989.

[37] S. N. Rogak and R. C. Flagan, "Coagulation of aerosol agglomerates in the transition regime," Journal of Colloid and Interface Science, vol. 151, no. 1, pp. 203-224, 1992.

[38] S. Modem, S. C. Garrick, M. R. Zachariah, and K. E. J. Lehtinen, "Direct numerical simulation of nanoparticle coagulation in a temporal mixing layer," in Proceedings of the 29th Symposium (International) on Combustion, pp. 1071-1077, The Combustion Institute, Pittsburgh, Pa, USA, 2002.

[39] S. C. Garrick and G. Wang, "Modeling and simulation of titanium dioxide nanoparticle synthesis with finite-rate sintering in planar jets," Journal of Nanoparticle Research, vol. 13, no. 3, pp. 973-984, 2011. 
[40] M. C. Heine and S. E. Pratsinis, "Polydispersity of primary particles in agglomerates made by coagulation and sintering," Journal of Aerosol Science, vol. 38, no. 1, pp. 17-38, 2007.

[41] R. W. MacCormack, "The effect of viscosity in hypervelocity impact catering," AIAA Paper 69-354, 1969.

[42] M. H. Carpenter, "A high-order compact numerical algorithm for supersonic flows," in Twelfth International Conference on Numerical Methods in Fluid Dynamics, K. W. Morton, Ed., vol. 371 of Lecture Notes in Physics, pp. 254-258, Springer, Berlin, Germany, 1990.

[43] D. H. Rudy and J. C. Strikwerda, "Boundary conditions for subsonic compressible navier-stokes calculations," Computers and Fluids, vol. 9, no. 3, pp. 327-338, 1981.

[44] P. Givi, "Filtered density function for subgrid scale modeling of turbulent combustion," AIAA Journal, vol. 44, no. 1, pp. 16-23, 2006.

[45] S. Modem and S. C. Garrick, "Nanoparticle coagulation in a temporal mixing layer mean and size-selected images," Journal of Visualization, vol. 6, no. 3, pp. 293-302, 2003.

[46] D. L. Wright, S. Yu, P. S. Kasibhatla et al., "Retrieval of aerosol properties from moments of the particle size distribution for kernels involving the step function: cloud droplet activation," Journal of Aerosol Science, vol. 33, no. 2, pp. 319-337, 2002.

[47] H. K. Kammler, R. Jossen, P. W. Morrison Jr., S. E. Pratsinis, and G. Beaucage, "The effect of external electric fields during flame synthesis of titania," Powder Technology, vol. 135-136, pp. 310$320,2003$.

[48] W. C. Hinds, Aerosol Technology: Properties, Behavior and Measurement of Air-Borne Particles, John Wiley \& Sons, New York, NY, USA, 2nd edition, 1999.

[49] N. Settumba and S. C. Garrick, "A comparison of diffusive transport in a moment method for nanoparticle coagulation," Journal of Aerosol Science, vol. 35, no. 1, pp. 93-101, 2004.

[50] S. E. Pratsinis, "Simultaneous nucleation, condensation, and coagulation in aerosol reactors," Journal of Colloid And Interface Science, vol. 124, no. 2, pp. 416-427, 1988.

[51] R. McGraw, "Description of aerosol dynamics by the quadrature method of moments," Aerosol Science and Technology, vol. 27, no. 2, pp. 255-265, 1997.

[52] J. Bai, Y.-H. Xu, and J.-P. Wang, "Cubic and spherical highmoment FeCo nanoparticles with narrow size distribution," IEEE Transactions on Magnetics, vol. 43, no. 7, pp. 3340-3342, 2007.

[53] A. Khorsand Zak, R. Razali, W. H. Abd Majid, and M. Darroudi, "Synthesis and characterization of a narrow size distribution of zinc oxide nanoparticles," International Journal of Nanomedicine, vol. 6, no. 1, pp. 1399-1403, 2011.

[54] M. Asemi and M. Ghanaatshoar, "Preparation of $\mathrm{CuCrO}_{2}$ nanoparticles with narrow size distribution by sol-gel method," Journal of Sol-Gel Science and Technology, vol. 70, no. 3, pp. 416421, 2014.

[55] A. M. Ahadi, O. Polonskyi, U. Schürmann, T. Strunskus, and F. Faupel, "Stable production of $\mathrm{TiO}_{\mathrm{x}}$ nanoparticles with narrow size distribution by reactive pulsed dc magnetron sputtering," Journal of Physics D: Applied Physics, vol. 48, no. 3, Article ID 035501, 2015.

[56] W. W. So, S. B. Park, K. J. Kim, C. J. Shin, and S. J. Moon, “The crystalline phase stability of titania particles prepared at room temperature by the sol-gel method," Journal of Materials Science, vol. 36, no. 17, pp. 4299-4305, 2001.
[57] A. Teleki, R. Wengeler, L. Wengeler, H. Nirschl, and S. E. Pratsinis, "Distinguishing between aggregates and agglomerates of flame-made $\mathrm{TiO}_{2}$ by high-pressure dispersion," Powder Technology, vol. 181, no. 3, pp. 292-300, 2008. 

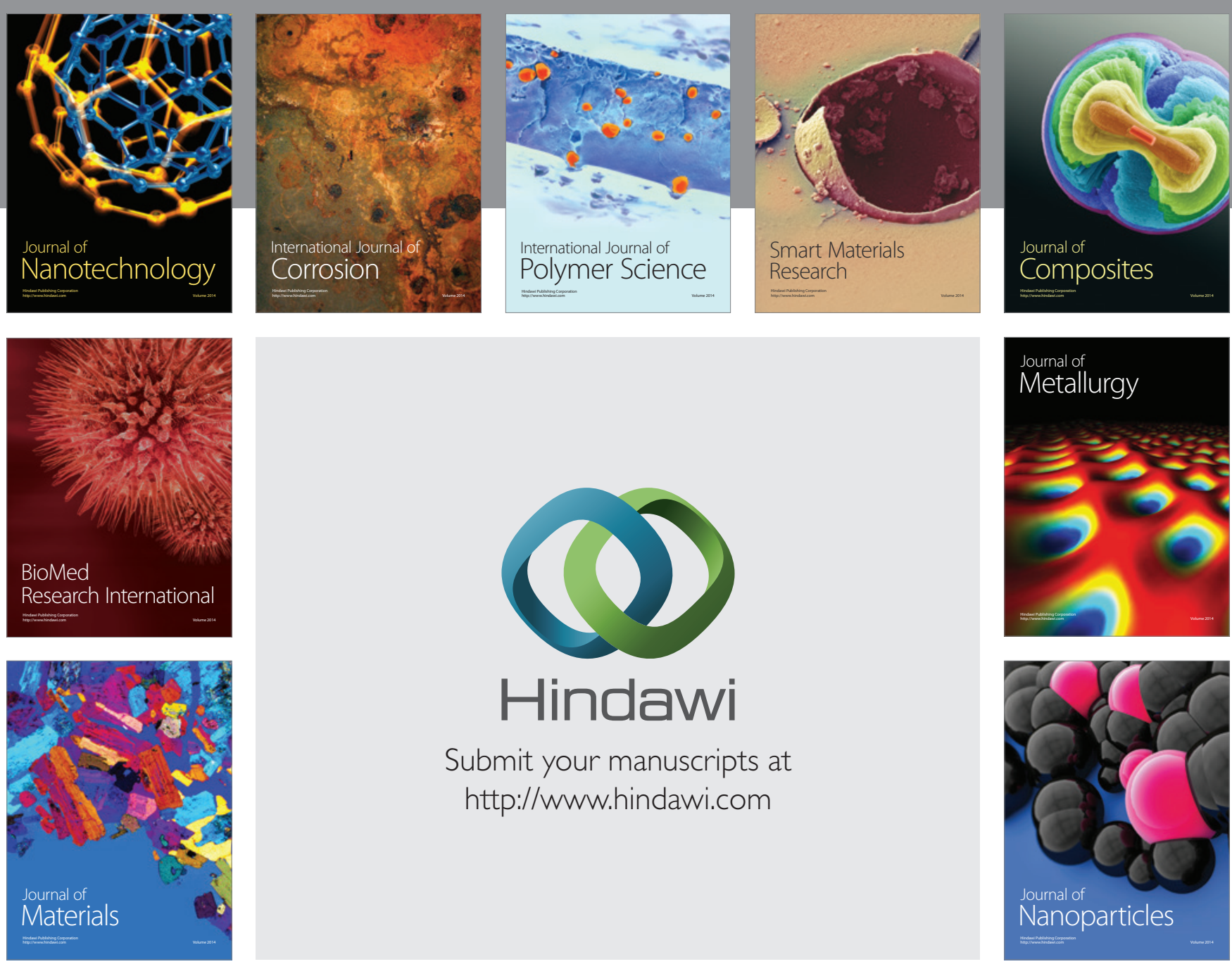

Submit your manuscripts at http://www.hindawi.com
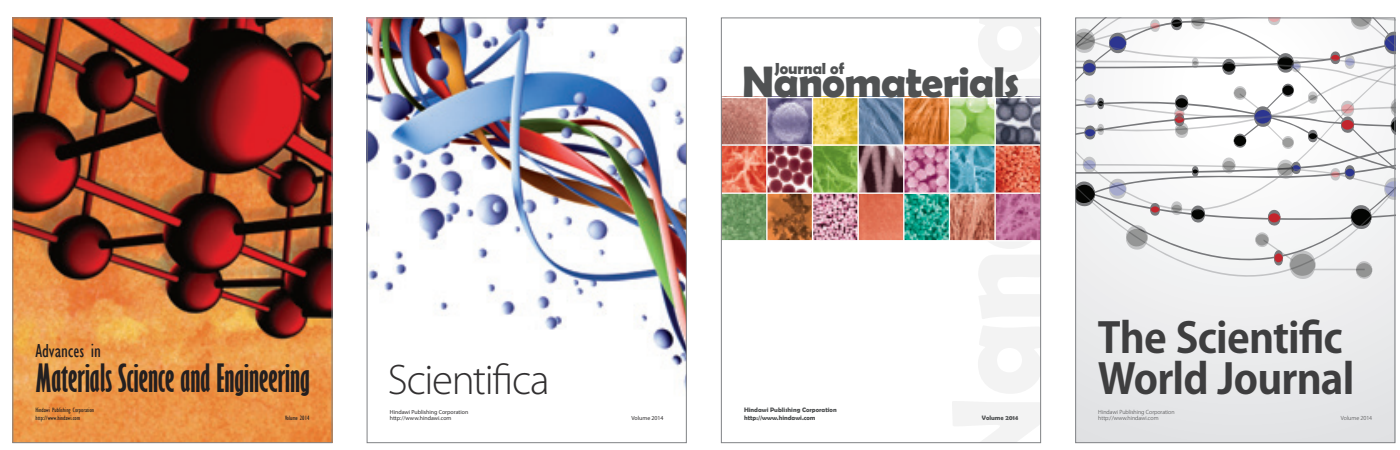

\section{The Scientific World Journal}
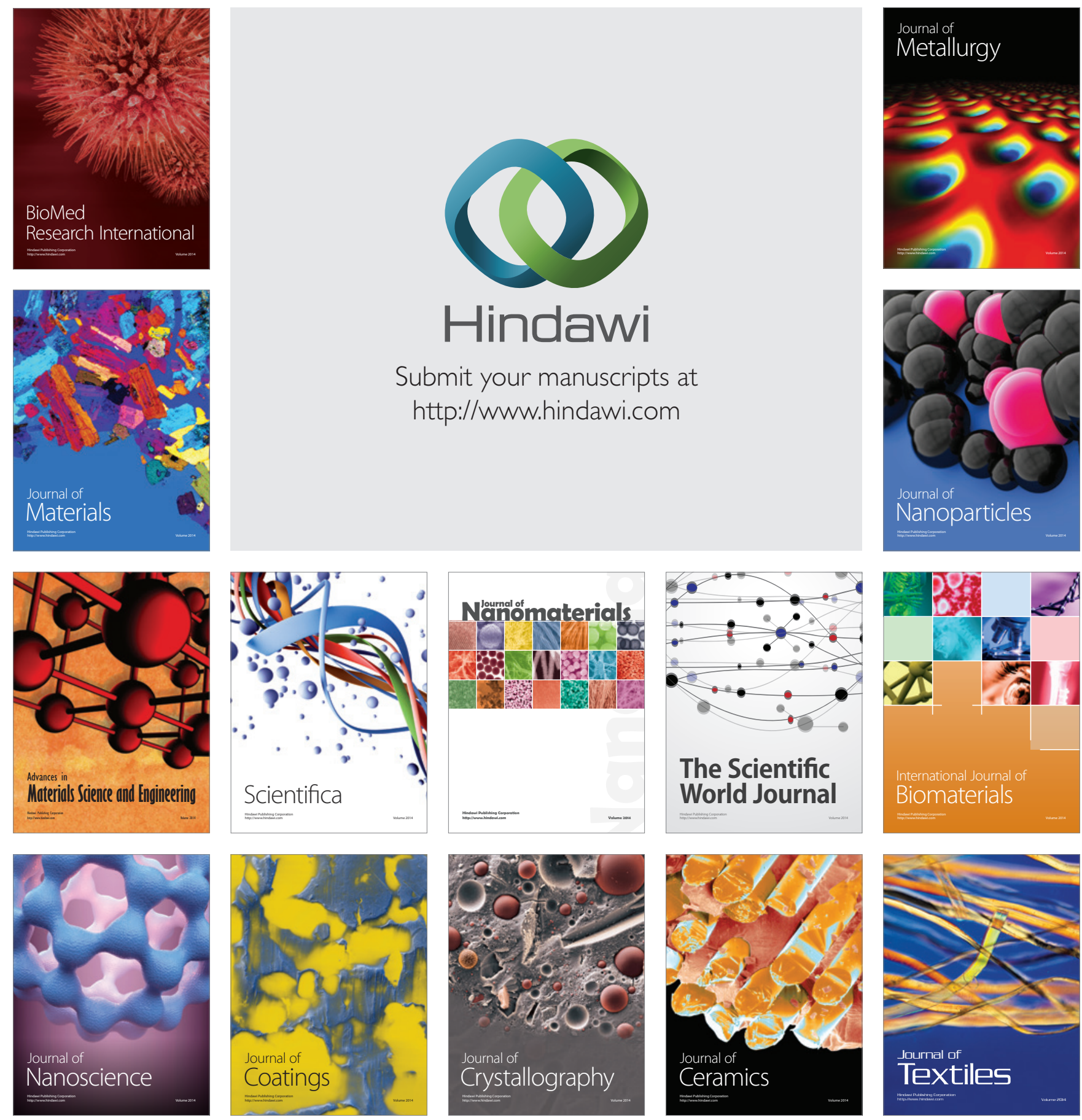